\title{
Análise Temporal de Índices Radiométricos no Município de Bebedouro, São Paulo
}

Temporal Analysis of Radiometric Index in the Municipality of Bebedouro, São Paulo

Análisis Temporal de los Índices Radiométricos en el Municipio de Bebedouro, São

Paulo

\author{
Glaucia Regina Santos \\ Mestre, UNESP, Brasil. \\ glauciaflorestal@gmail.com
}

Ana Paula Leite

Mestranda, UNESP, Brasil. anapaulinhaleite@gmail.com

Victor Costa Leda

Doutorando, UNESP, Brasil. victorleda@gmail.com 


\section{RESUMO}

Nas sociedades modernas o sensoriamento remoto surge para fornecer informações temporais de extensas áreas com alto grau de detalhamento, a fim de analisar e monitorar as modificações de uso do solo. Desse modo, o objetivo deste trabalho foi verificar as modificações no uso do solo no município de Bebedouro - SP em um período de 20 anos e entender quais fatores podem se relacionar com a expansão do município. Foram utilizadas imagens em épocas distintas (1995 e 2015) para toda a extensão da cidade. Os índices utilizados neste trabalho foram o SAVI, MSAVI2 e NDBI. Observou-se que as manchas urbanas presente nos índices apresentam-se inseridas na mesma classe de solo exposto. Ocorreu maior amplitude geral do índice NDBI, no ano de 1995 e 2015 . Ao comparar o SAVI do MSAVI2, observa-se que MSAVI2 apresenta maior grau de detalhamento. A amostragem temporal nos indica que no município ocorreu um aumento de área com biomassa vegetal, isto porque houve um aumento na média dos valores dos pixels dos índices SAVI e MSAVI2 das imagens processadas, e um aumento de áreas urbanas e estéreis, caracterizada pelo aumento na média dos valores dos pixels do índice NDBI. O município apresentou um incremento em área urbana de $25,41 \%$. Pode-se associar que o crescimento espacial urbano relaciona-se com o aumento do número de habitantes. Porém, tanto os valores de expansão espacial como populacional apresentaram valores reduzido quando comparado com cidades de economia bem desenvolvida.

PALAVRAS-CHAVE: Sensoriamento remoto. Expansão urbana. Processamento de imagens.

\section{ABSTRACT}

In modern societies, the remote sensing provides temporal information in wide areas with high detail level, and to analyze and monitoring the modification of soil use. This way, the aim of this work was to verify the modification in soil use in the municipality of Bebedouro - SP, in a 20 years period and understand which factors can be related with the city expansion. It was used images in distinct periods (1995 and 2015) for all the city extension. The indexes used in this work were: SAVI (Soil Adjusted Vegetation Index), MSAVI2 (Modification Soil Adjusted Vegetation Index 2) and NDBI (Normalized Difference Built-up Index). It was observed that the urban area presented in the indexes are shown inserted in the same exposed soil class. Comparing SAVI and MSAVI2, it was observed that MSAVI2 shows higher detail level. The temporal sampling indicates that in the city occurred a vegetal biomass area growing, due to an increase of the pixels values mean in SAVI an MSAVI2 of processed images, and an increase of urban and sterile areas, characterized by the increasing in NDBI pixels values mean. The city showed an urban area increment of $25,41 \%$. This spatial urban growing is related to the inhabitants' number growth. However, even the spatial expansion as the population showed reduced when compared with well-developed economy cities.

KEYWORDS: Remote sensing. Urban expansion. Images processing.

\section{RESUMEN}

En las sociedades modernas la teledetección parece proporcionar informaciones temporales de grandes áreas con un alto grado de detalle, con el fin de analizar y supervisar los cambios de uso de la tierra. Así, el objetivo de este trabajo fue verificar los cambios en la tierra la ciudad de Bebedouro-SP en un período de 20 años y comprender qué factores pueden relacionarse con la expansión urbana. Imágenes fueron utilizadas en diferentes épocas del año (1995 y 2015) para la longitud entera del Municipio. Los índices utilizados en este trabajo fueron la SAVI (índice suelo-ajustado de la vegetación), MSAVI2 (modificaci\& oacute;n del índice de vegetación ajustado de suelo 2) y NDBI (normalizado índice de diferencia de las zonas edificadas). Se observó que la mancha urbana en los índices se incluyen en la misma clase de suelo expuesto. Mayor magnitud ocurrió índice NDBI general, en el año 1995 y 2015. Al comparar la SAVI de la MSAVI2, MSAVI2 presenta un mayor grado de detalle. El muestreo temporal nos indica que em la ciudad ocurrió um aumento de áreas com biomasa vegetal, debido al aumento del promedio de valores de los pixels de los índices SAVI y MSAV12 de las imagenes procesadas y un aumento de áreas urbanas y estériles, caracterizadas por el aumento em el promedio de los valores de los pixels del indice NDBI. El municipio presentó un incremento en área urbana del $25,41 \%$.Se puede asociar que el crecimiento espacial urbano se relaciona con el aumento del número de habitantes. Sin embargo, tanto los valores de expansión espacial como poblacional presentaron valores reducido cuando comparado con ciudades de economía bien desarrollada.

Palabras clave: Detección remota. Expansión urbana. Procesamiento de imágenes. 


\section{INTRODUÇÃO}

A constante expansão urbana tem sido motivo de muita preocupação nas sociedades modernas ( $\mathrm{Xu}$ et al., 2010). Isto porque o crescimento desorganizado e espontâneo das cidades é acompanhado de um número maior de veículos, indústrias, rodovias, ruas asfaltadas, prédios, redução de áreas com vegetação e dentre outros fatores, que podem trazer prejuízos ao meio ambiente e ao bem estar humano, como por exemplo, maior impermeabilidade do solo e elevar a temperatura da superfície (Carvalho et al., 2015). Esses resultados se relacionam com o crescimento da população, fatores econômicos, proximidade dos recursos e as comodidades básicas como internet, água e esgoto encanado (Hegazy; Kaloop, 2016).

Neste contexto, o sensoriamento remoto surge para fornecer informações temporais de extensas áreas com alto grau de detalhamento, assim como analisar e monitorar as modificações de uso do solo e expansão urbana de forma mais rápida e rentável (Deng et al., 2009). Dentre as técnicas existentes, pode-se aplicar o sensoriamento remoto no monitoramento ambiental, avaliação do estado da vegetação, observação da infraestrutura de rodovias e monitoramento do aumento de desertos (Mei et al., 2016).

Cabe ressaltar, que mapas sobre áreas urbanas apresentam uma grande variedade de informações como casas, apartamentos, indústrias, ruas, rodovias e áreas de lazer, intercalados com espaços vegetais naturais, o que torna o mapeamento difícil (García; Pérez, 2016). Por isso, há uma grande variedade de índices sobre mudanças no uso do solo específicos para cada localidade, como é o caso do NDBI (Normalized Difference Built-up Index) usado para discriminar áreas urbanas e construídas (Chen et al., 2006), SAVI (Soil Adjusted Vegetation Index) que é uma adaptação do NDVI (Normalized Difference Vegetation Index), utilizado em estudos de vegetação onde há uma influência grande do solo (García; Pérez, 2016) e o índice MSAVI2 (segunda modificação do SAVI) que amplia o alcance dinâmico do sinal da vegetação e minimiza ainda mais os efeitos do solo (Qi et al., 1994).

\section{OBJETIVOS}

Verificar as modificações no uso do solo, a partir da aplicação dos índices SAVI, MSAVI2 e NDBI em imagens de satélite, em dois períodos diferentes. E entender quais fatores podem se relacionar com a velocidade do aumento espacial urbano do município, no período de 20 anos.

\section{METODOLOGIA}

\section{1 Descrição e histórico da área de estudo}

A área de estudo situa-se na cidade de Bebedouro, localizada na região noroeste do estado de São Paulo, nas coordenadas 20 53'16" S e 48 28'11" W, altitude de $601 \mathrm{~m}$, clima subtropical com inverno moderado seco e verão quente e chuvoso, temperatura média $23,5 \circ \mathrm{C}$ e precipitação anual de 1.522 mm (Figueiredo et al., 2002). Na Figura 1 pode-se visualizar a localização da cidade 
Figura 1: Localização do município de Bebedouro-SP

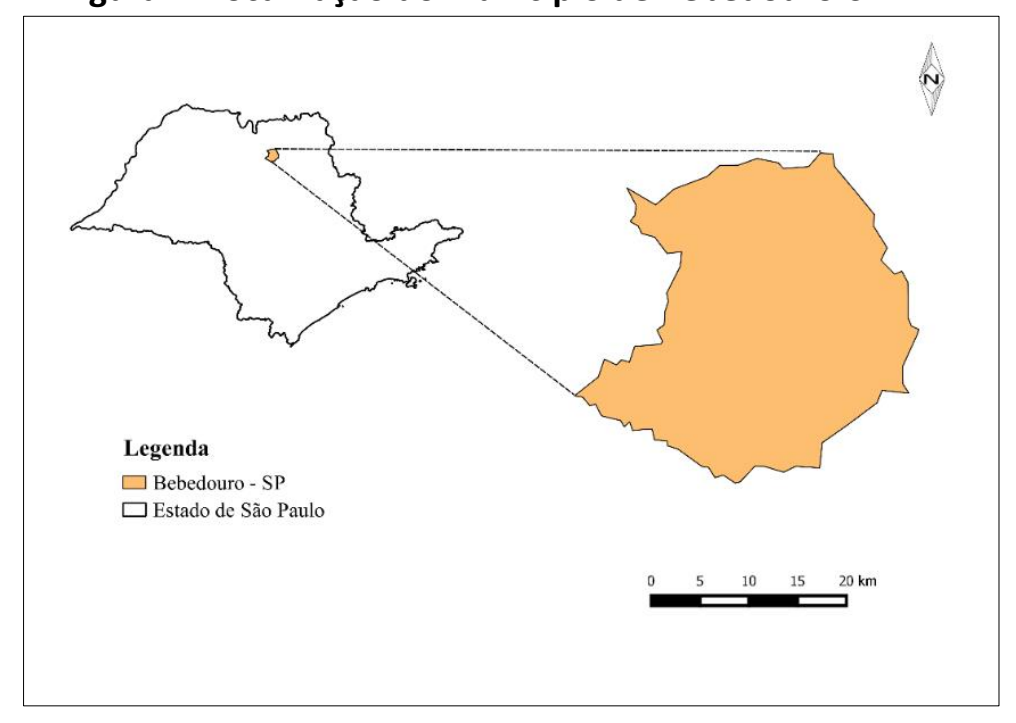

A cidade surgiu por ser rota de boiadeiros e tropeiros que paravam em um córrego existente no município para alimentar e saciar a sede dos animais de carga que acompanhavam a viagem (IBGE, 2016). Atualmente o município apresenta uma população estimada de 77,67 mil habitantes, uma área total de $683,19 \mathrm{~km}^{2}$ e densidade demográfica de 109,81 hab/ $\mathrm{km}^{2}$ (IBGE, 2016).

Com a crise de 1929 o município substituiu a cafeicultura pela citricultura e por meio desta atividade, apresentou um rápido crescimento populacional, porém com o fim do ciclo da laranja, o foco econômico da cidade se transferiu para o setor de comércio, serviços e à canade-açúcar (Comunicação pessoal).

\section{2 Metodologia de trabalho}

Para a realização do estudo foram utilizadas imagens em épocas distintas (1995 e 2015) do Sensor Thematic Mapper (TM) do satélite Landsat-5 e o Sensor Operational Land Imager (OLI) do satélite Landsat-8 para toda a extensão da cidade de Bebedouro - SP. Optou-se pelo uso de duas imagens para se verificar a expansão da cidade e os diferentes usos do solo com o passar dos anos. Essas imagens foram obtidas da base de dados da U.S. Geological Survey (USGS) disponível em (http://earthexplorer.usgs.gov/). Os dados e os mapas finais foram processados por meio do programa Qgis 2.8.3. As imagens foram obtidas em um período seco, e correspondem a órbita/ponto 221/74, com data de passagem no dia 29 (vinte e nove) de agosto de 1995 e 21 (vinte e um) setembro de 2015. Assim como no trabalho do Carvalho et al (2015) para efetuar a confecção das imagens na cor normal (RGB) e a análise dos índices, foram obtidas as bandas espectrais da faixa do visível e da faixa do infravermelho. Após obtidas as imagens, foi efetuado a correção atmosférica destas para amenizar os efeitos dos gases da atmosfera. Posteriormente as bandas foram reprojetadas para o hemisfério sul porque as imagens obtidas pela base de dados USGS apresentam-se no hemisfério norte. 
Os índices utilizados neste trabalho foram o SAVI (Soil-Adjusted Vegetation Index), MSAVI2 (segunda modificação do SAVI) e o NDBI (Normalized Difference Built-up Index). O uso dos índices auxilia na comparação e verificação da precisão das informações obtidas (García; Pérez, 2016). O índice NDBI, desenvolvido por Zha et al.(2003), é útil na obtenção de informações de áreas urbanas e construídas (Chen et al., 2006), na Equação 1 pode-se visualizar sua fórmula:

$$
N D B I=\frac{S W I R-N I R}{S W I R+N I R}
$$

Sendo SWIR referente a banda do infravermelho médio e NIR a banda do infravermelho próximo. Esse índice na maioria das vezes baseia-se na maior reflectância na faixa do SWIR do que do NIR de áreas urbanizadas, porém em locais com vegetação seca, pode haver maior reflectância do SWIR resultando em valores positivos de NDBI para esses locais (Xu, 2007).

Cabe ressaltar que o índice NDBI varia de -1 a 1 , e os valores positivos representam áreas construídas/impermeabilizadas e os valores negativos correspondem as áreas com cobertura vegetal (Silva et al., 2014), porém a presença de vegetação seca e água com elevada concentração de material em suspensão também podem elevar os valores positivos para o $\operatorname{NDBI}(X u, 2007)$.

O NDVI é um índice amplamente utilizado em estudos de sensoriamento remoto, porém como o presente estudo relaciona-se com uma área urbanizada de menor cobertura vegetal, optouse pelo uso do SAVI (Xu, 2007). Esse índice é uma adaptação do NDVI sendo utilizado em locais onde há influência significativa do solo (García; Pérez, 2016). Cabe ressaltar, que o índice SAVI pode ser usado em áreas com baixa cobertura vegetal, em torno de $15 \%$, enquanto que o NDVI pode ser usado de forma eficaz em áreas com densidade vegetal acima de $30 \%$. (Xu, 2007). Na Equação 2 pode-se visualizar a fórmula do SAVI.

$$
S A V I=\frac{N I R-R E D}{N I R+R E D+L}(1+L)
$$

Onde NIR é a banda do infravermelho próximo, RED é a banda do vermelho e $L$ é o fator de ajuste para presença de vegetação. Para cobertura vegetal de menor densidade o fator $L$ é 1,0 ; para cobertura densidade vegetal intermediária o fator será 0,5 , e para altas densidades, fator 0,25; neste trabalho utilizou-se o ajuste $L$ de 0,5. Já o índice MSAVI é proveniente de uma modificação do índice SAVI porque ele substitui o fator de ajuste manual (L) encontrada no SAVI por um auto ajuste do L. Esse fator, apesar de não estar presente na segunda modificação do SAVI, foi utilizado na derivação da fórmula do MSAVI2. Portanto, a grande diferença do índice SAVI para o MSAVI2, é que no índice SAVI necessita-se de um conhecimento prévio da vegetação para a definição do valor do fator $L$, enquanto que no MSAVI2 esse fator é auto ajustável (Qi et al., 1994).

$$
\text { MSAVI2 }=\frac{2 \cdot \mathrm{NIR}+1-\sqrt{(2 \cdot \mathrm{NIR}+1)^{2}-8 \cdot(\mathrm{NIR}-\mathrm{RED})}}{2}
$$


Onde o NIR representa a banda do infravermelho próximo, RED corresponde a banda do vermelho. Esse índice foi proposto por Ql et al. (1994) e os valores inserem-se em um intervalo de -1 a +1 (Arenas-Toledo; Espiphanio, 2007).

\section{RESULTADOS}

As imagens processadas nos anos de 1995 (Landsat 5) e 2015 (Landsat 8) referentes aos índices SAVI, MSAVI2 e NDVI podem ser visualizadas nas Figuras 2,3 e 4. Essas imagens fornecem informações sobre os cenários encontrados em cada período.

Podemos observar mais de um uso do solo por classe nos dois períodos estudados dos índices SAVI e MSAVI2. No caso, a mancha urbana presente nos índices está inserida na mesma classe de solo exposto (classe em vermelho), a classe azul está relacionada com áreas de agricultura de maior densidade e área nativa e as cores laranja, amarelo e verde caracterizam os vários estágios de crescimento vegetal e ocupação do solo.

Figura 2: Mapas do índice SAVI

1995

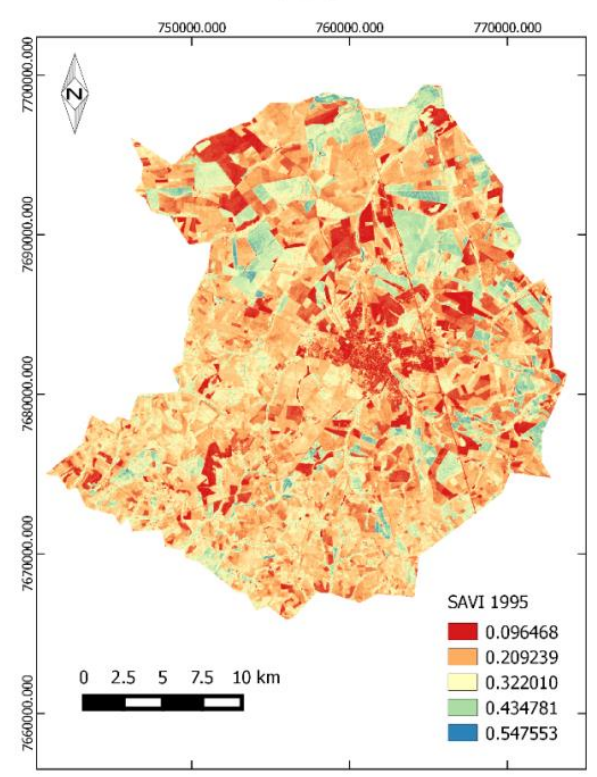

2015

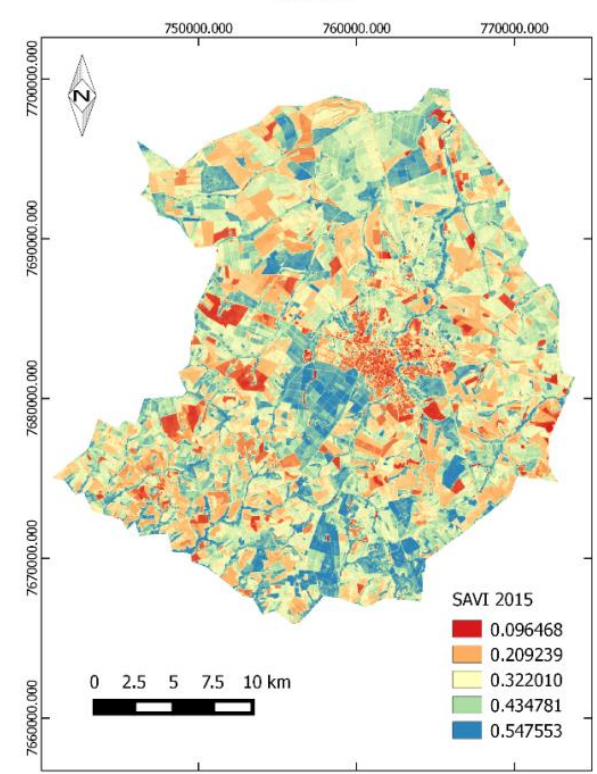

Observa-se nas Figuras 2 e 3, das imagens processadas dos índices SAVI e MSAVI2, que o índice MSAVI2 realçou mais as características do solo do que o SAVI, isto porque nota-se um tom mais avermelhado na imagem de 1995 e mais amarelado na imagem do ano de 2015 do MSAVI2 quando comparada com o SAVI. 
Figura 3: Mapas do índice MSAVI2

\section{5}

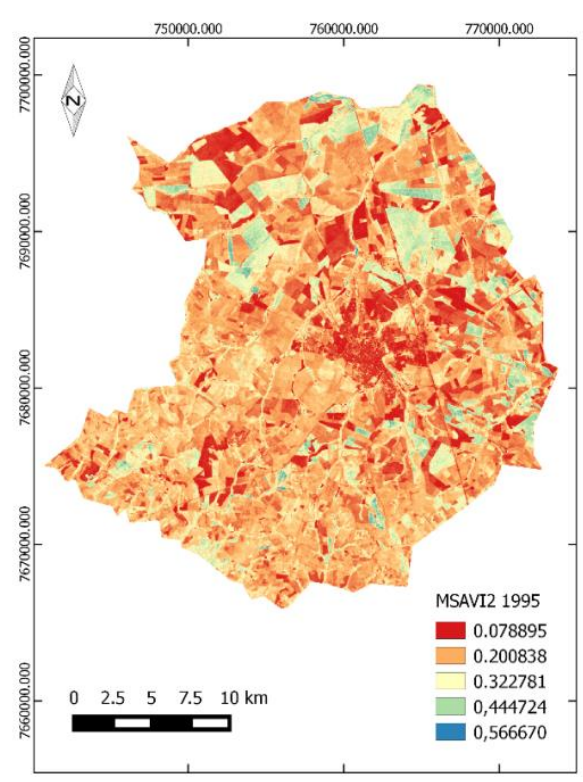

2015

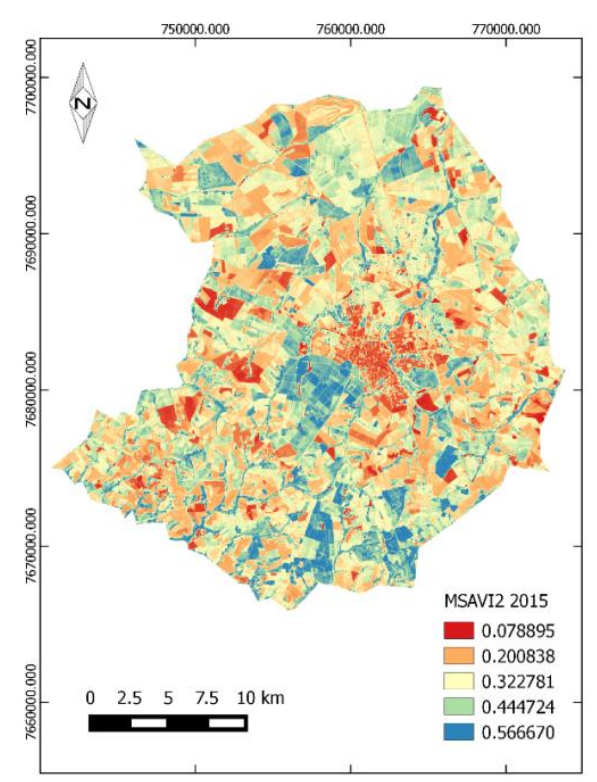

O índice NDBI foi desenvolvido para detectar áreas construídas, com elevada resposta nas bandas do infravermelho médio do que o infravermelho próximo (García; Pérez, 2016).Este índice seguiu o mesmo padrão dos SAVI e MSAVI2 porque não conseguiu discriminar separadamente as áreas construídas de solo seco nu ou solo exposto (Figura 4). Esse mesmo padrão ocorreu nos trabalhos de Rafael (2014) e Weihua et al. (2006), segundo estes últimos autores a utilização do NDBI deve focar-se em regiões com alta cobertura vegetal e em períodos de floração, pois assim pode-se determinar com maior precisão as áreas construídas. 
Figura 4: Mapas do índice NDBI

1995

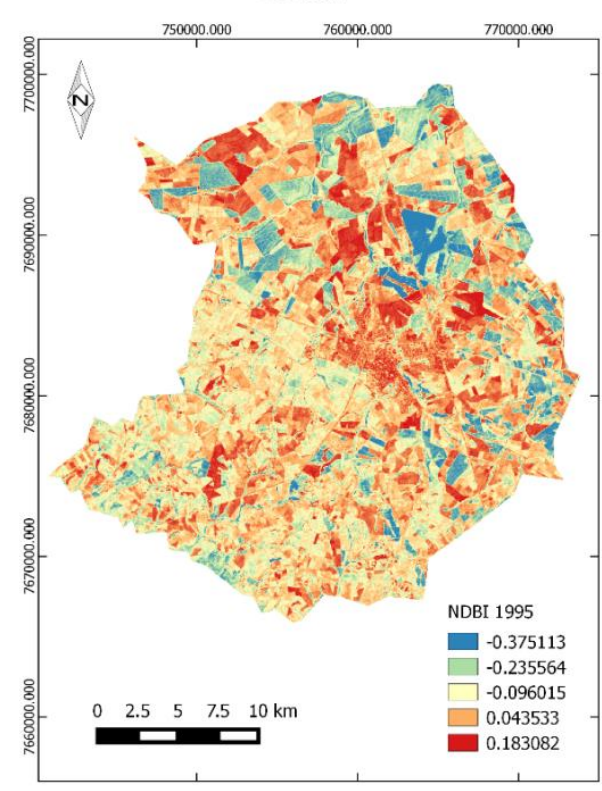

2015

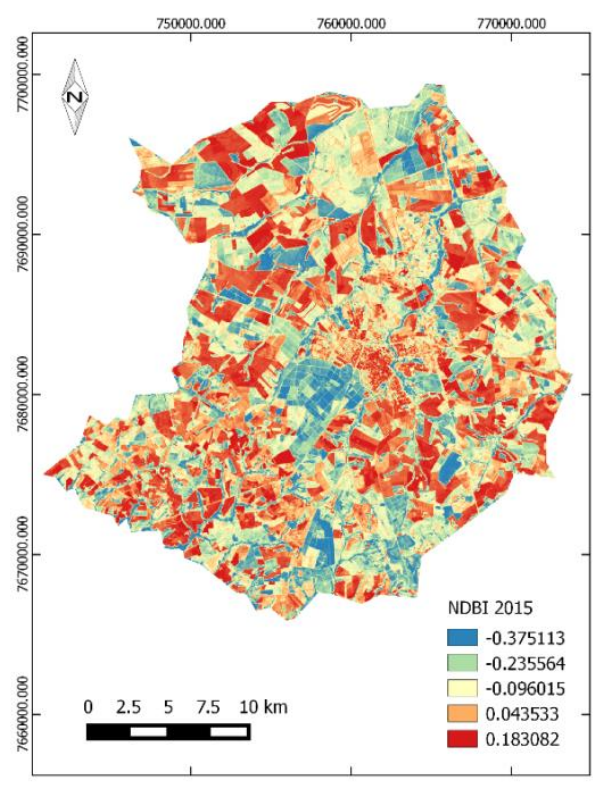

Segundo Xu (2007) a maior amplitude do índice reflete um maior detalhamento das áreas vegetadas em contraste com o solo exposto e/ou impermeabilizado. Neste caso, a Tabela 2 expõe a amplitude dos índices em 1995 e 2015. Pode-se notar a maior amplitude do índice NDBI no ano de 1995 e 2015 e, porém ao comparar o SAVI do MSAVI2, observa-se que MSAVI2 apresenta maior grau de detalhamento.

Tabela 1: Amplitude dos índices SAVI, MSAVI2 e NDVI no ano de 1995 e 2015

19952015

\begin{tabular}{|c|c|c|c|c|c|c|}
\hline Índices & Mínimo & Máximo & Diferença & Mínimo & Máximo & Diferença \\
\hline SAVI & 0,0755 & 0,4057 & 0,3302 & 0,1564 & 0,5567 & 0,4003 \\
\hline MSAVI2 & 0,0788 & 0,4346 & 0,3558 & 0,1140 & 0,5666 & 0,4526 \\
\hline NDBI & $-0,3799$ & 0,1030 & 0,4829 & $-0,3711$ & 0,1648 & 0,5359 \\
\hline
\end{tabular}

No ano de 1995 para 2015, para os índices SAVI, MSAVI2 e NDBI os valores da média dos pixels foram respectivamente 0,252 e 0,347; 0,221 e 0,329; 0,054 e 0,061. A amostragem temporal nos indica que no município de Bebedouro ocorreu um aumento de área com biomassa vegetal, isto porque houve um aumento na média dos valores dos pixels dos índices SAVI e MSAVI2 das imagens processadas, assim como um aumento de áreas urbanas e estéreis, caracterizada pelo aumento na média dos valores dos pixels do índice NDBI.

As imagens processadas obtidas pelo SAVI e MSAVI2 relacionam-se com índices espectrais de vegetação (Oliveira et al. 2009), já a imagem do índice NDBI é composta por pixels relacionados com áreas construídas e estéreis (Silva et al., 2014; Jensen, 2009). 
Segundo Rafael (2014) o processo de urbanização apresenta inicialmente a substituição da vegetação nativa por um solo semi exposto ou exposto para um futuro uso tanto na construção civil como para o uso na agricultura. Nota-se em alguns pontos, a retirada da vegetação para a substituição por áreas agrícolas, portanto, é possível que este cenário de maior cobertura vegetal relaciona-se com a mudança no uso do solo para a agricultura. 0 incremento em área urbana foi determinado por meio da diferença de área dos polígonos, utilizando-se imagens dos índices e de diferentes composições de bandas. A Tabela 3 apresenta o incremento em área urbana do município, foi obtida uma expansão urbana de $25,41 \%$ em 20 anos.

Tabela 2: Tabela do crescimento da área urbana em $\mathrm{m}^{2}$

\begin{tabular}{cccc}
\hline Ano & Área urbana $\left(\mathrm{m}^{2}\right)$ & Incremento $\left(\mathbf{m}^{2}\right)$ & Crescimento urbano (\%) \\
\hline 1995 & 1622,25 & - & - \\
2015 & 2175,07 & 552,82 & 25,41 \\
\hline
\end{tabular}

Pode-se associar que o crescimento espacial urbano verificado relaciona-se com o aumento do número de habitantes, segundo informações do IBGE (2016), em 1996 o município de Bebedouro apresentou o total de 72.233 hab. e em 2015 o número de 77.627 hab., um acréscimo de 5.394 hab. em 19 anos, portanto um aumento populacional de 6,94\%. Cabe ressaltar que a cidade apresenta atualmente uma economia voltada principalmente para o mercado de serviços do que a indústria e agropecuária (IBGE, 2016).

Porém, tanto os valores de expansão espacial como populacional apresentaram valores reduzido quando comparado com cidades de economia bem desenvolvida, como pode ser observado nos trabalhos de Deng et al. (2009), Hegazy e Kaloop (2015) e Xu et al. (2010).

\section{CONCLUSÕES}

O uso dos índices SAVI, MSAVI2 e NDBI foi satisfatório no auxílio da interpretação das imagens processadas.

No período de 20 anos, foi constatado um incremento espacial urbano e populacional, porém com uma velocidade menor quando comparada com grandes cidades.

O sensoriamento remoto é uma boa ferramenta para analisar a intensidade e evolução da expansão urbana a partir de imagens em períodos diferentes.

\section{AGRADECIMENTO}

Ao grupo GEPAG (Grupo de Estudo e Pesquisas Agrárias Georreferenciadas) da UNESP de Botucatu e à Prof. Célia Regina Lopes Zimback. 


\section{REFERÊNCIAS BIBLIOGRÁFICAS}

Arenas-Toledo, J. M. Epiphanio, J. C. N. Comportamento de Índices de Vegetação a partir de três sensores orbitais: estudo de caso no município de São Borja (RS). In: Anais XIII Simpósio Brasileiro de Sensoriamento Remoto, 13 (SBSR), Florianópolis, p.741-748, 2007.

Carvalho, I. M.; Cunha, A. A.; Souza, F. A.; Pedrassoli, J. C. Integração entre SR e SIG para a modelagem e o gerenciamento de dados espaço temporais de temperatura de superfície utilizando imagens do sensor ETM do LANDSAT 7. In: Anais XVII Simpósio Brasileiro de Sensoriamento Remoto, 17 (SBSR), João Pessoa, PB, 2015.

Chen, X. L.; Zhao, H. M.; Li, P. X.; Yin, Z. Y. Remote sensing image-based analysis of the relationship between urban heat island and land use/cover changes. Remote Sensing of Environment, v. 104, n. 2, p. 133-146, 2006.

Deng, J. S.; Wang, K.; Hong, Y.; Qi, J. G. Spatio-temporal dynamics and evolution of land use change and landscape pattern in response to rapid urbanization. Landscape and Urban Planning,v. 92, p. 187-198, set., 2009.

Figueiredo, J. O.; Stuchi, E. S. ; Donadio, L. C. ; Sobrinho, J. T. ; Laranjeira, F. F. ; PIO, R. M. ; Sempionato, O. R. Porta-enxertos para a Lima-ácida-'tahiti' na região de Bebedouro, SP. Revista Brasileira Fruticicultura, Jaboticabal - SP, v. 24, n. 1, p. 155-159, abril 2002.

García, P.; Pérez, E. Mapping of soil sealing by vegetation indexes and built-up index: A case study in Madrid (Spain). Geoderma, v. 268, p.100-107, abril, 2016.

Hegazy, I. R.; Kaloop, M. R. Monitoring urban growth and land use change detection with GIS and remote sensing techniques in Daqahlia governorate Egypt. International Journal of Sustainable Built Environment, v. 4, p.117-124, jun., 2015.

IBGE - Instituto Brasileiro de Geografia e Estatística. Disponível em: http://www.cidades.ibge.gov.br/xtras/perfil.php?lang=\&codmun=350610\&search=saopaulo|bebedouro. Acesso em: 16 de abr. 2016.

Jensen, J. R. Sensoriamento remoto do ambiente: Uma perspectiva em recursos terrestres. Editora Parêntese, São José dos Campos, SP, p. 598, 2009.

Mei, A; Manzo, C.; Fontinovo, G.; Bassani, C.; Allegrini, A.; Petracchini, F. . Assessment of land cover changes in Lampedusa Island (Italy) using Landsat TM and OLI data. Journal of African Earth Sciences, 2015. DOI: 10.1016/j.jafrearsci.2015.05.014

Oliveira, W. M.; Chavez, I. B.; Lima, E. R. V. Índices espectrais de vegetação de caatinga em um Neossolo Litólico do semi-árido paraibano. In: Anais XIV Simpósio Brasileiro de Sensoriamento Remoto. p. 21032110, abril, 2009.

Qi, J.; Chehbouni, A.; Huete, A. R.; Kerr, Y. H.; Sorooshian, S. A modified soil adjusted vegetation index. Remote Sensingof Environment, v. 48, p. 119-126, 1994.

Rafael, R. A. Relação entre ilhas de calor urbano e mudanças no uso da terra: Um estudo de caso para Manaus - AM. 2014. 60p. Dissertação (Mestrado em Meteorologia). Universidade Federal de Campina Grande - UFCG. 2014. 
Silva, F. M.; Costa Junior, N. P.; Lima, Z. M. C. Avaliação microclimática de Natal/RN através de técnicas de sensoriamento remoto: uma contribuição aos estudos do clima urbano. Sociedade e Território, Natal, v. 26, no 2, p. 163 - 180, jul./dez., 2014.

Weihua, P.; Hui, C.; Chungui, Z. Study On The Methods Of Extracting Urban Land-Use Information Based On Landsat Imagery - Taking Quanzhou City For Example. The International Societyof Optical Engineering. Bellingham, jan., 2006. DOI: 10.1117/12.673665

$\mathrm{Xu}, \mathrm{H}$. Extraction of Urban Built-up Land Features from Landsat Imagery Using a Thematic oriented Index Combination Technique. Photogrammetric Engineering \& Remote Sensing, v. 73, n. 12, p. 1381-1391, dez., 2007.

Xu, Y.; Qin, Z.; Wan, H. J. Spatialand Temporal Dynamics of Urban Heatls landand Their Relationship with Land Cover Changes in Urbanization Process: A Case Study in Suzhou, China. Journal of the Indian Societyof Remote Sensing, v.38, n.4, p.654-663, dez., 2010.

Zha, Y.; Gao, J. Ni, J. Use of normalized difference built-up index in automatically mapping urban areas from TM imagery, International Journal of Remote Sensing, vol. 24, p. 583-594, 2003. 